\title{
Analysis of Trends of Sleep-Related Breathing Disorders in Patients with Systemic Essential Hypertension
}

\author{
Santosh Kumar Sinha1, Anand Kumar2, Avinash Jain'2, Ramesh Thakur1, \\ Chandra Mohan Varma1, Vinay Krishna1, Avdhesh Kumar ${ }^{2}$, Nirlep Gajiwala ${ }^{3}$, \\ Ashok Thakkar ${ }^{3}$ \\ ${ }^{1}$ L.P.S. Institute of Cardiology, G.S.V.M. Medical College, Kanpur, India \\ ${ }^{2}$ M.L. Chest Hospital, G.S.V.M. Medical College, Kanpur, India \\ ${ }^{3}$ Sahajanand Medical Technologies Pvt. Ltd., Surat, India \\ Email: fionasan@rediffmail.com
}

Received 16 July 2015; accepted 10 August 2015; published 13 August 2015

Copyright (C) 2015 by authors and Scientific Research Publishing Inc.

This work is licensed under the Creative Commons Attribution International License (CC BY). http://creativecommons.org/licenses/by/4.0/

\section{Open Access}

\section{Abstract}

Background: Studies have revealed a strong link between sleep-related breathing disorders and essential hypertension. However, the trends of sleep-disordered breathing in hypertensive patients have not been analyzed frequently. Methods: In this prospective, single-center and observational study, random patients with systemic essential hypertension, who were visiting GSVM Medical College, Kanpur, India between December 2012 and July 2014, were selected. After obtaining informed consent, patients were analyzed for symptoms of sleep-disordered breathing and Epworth Sleepiness Scale. Subsequently, all patients underwent overnight polysomnography to estimate the events of apnea and hypopnea. Observed data were compared between stage I and stage II hypertensive patients. Results: A total of 51 patients (age: $45.7 \pm 13.43$ years), comprising 36 stage I hypertensives and 15 stage II hypertensives, were enrolled in the study. About $47 \%$ of these patients displayed no symptoms of sleep-disordered breathing. Compared to stage I hypertensives, higher percentage of stage II hypertensives reported excessive day time sleepiness. Sleep latency time and sleep architecture were comparable between stage I and stage II hypertensives. Stage II hypertensives displayed shorter total sleep time and higher snore time than stage I hypertensives. Apnea Hypopnea Index (AHI) values increased with the severity of hypertension. Nocturnal oxygen desaturation was present in $\mathbf{2 5 . 0 \%}$ and $\mathbf{5 3 . 3 \%}$ of stage I and II hypertensives respectively. Mean lowest oxygen saturation was lower and the mean C-reactive protein levels were higher in stage II hypertensives as compared to stage I hypertensives. The mean C-reactive protein levels increased with increasing AHI scores, while the mean lowest oxygen saturation decreased with increasing AHI scores. Conclusion: Almost half of the hypertensives patients reported no symptoms of sleep-disordered breathing. Sleep-disordered breathing was more common and was 
more severe in majority of patients with stage II hypertension as compared to that in patients with stage I hypertension.

\title{
Keywords
}

\author{
Apnea, Hypertension, Polysomnography, Sleep Disorders
}

\section{Introduction}

Sleep-related breathing disorder, also called as sleep-disordered breathing, is estimated to affect a substantial proportion of adult population. It is a devastating condition characterized by apnea and hypopnea during sleep [1]. Sleep-related breathing disorders tend to alter sleep architecture and duration, resulting in daytime symptoms, signs, or systematic dysfunction. Earlier it has been reported that depriving healthy subjects of sleep may cause temporary increase in blood pressure levels and sympathetic nervous system activity. Further, prolonged deprivation of sleep may intensify the exposure to elevated 24-hour blood pressure and heart rate, increased sympathetic nervous system activity, and increased salt retention [2]. Hence, sleep-related breathing disorders are suggested to be an individual risk factor for the development of hypertension. Studies have revealed a significant association between sleep-related breathing disorders and systemic hypertension in middle aged and older individuals of different ethnicity [3]. According to a report, about $50 \%$ of patients with essential hypertension have obstructive sleep apnea and another $40 \%$ of these patients are habitual snorers without obstructive sleep apnea. It has also been shown that effective management of obstructive sleep apnea leads to reduction in elevated blood pressure in such patients [4]. Despite a strong link between sleep disordered breathing and essential hypertension [2] [3], limited studies have been conducted to evaluate sleep-related breathing disorders in systemic essential hypertensive patients. We believe that such studies will help in identifying and improving the role of sleep-related breathing disorders in hypertension management. In this regard, we conducted the present study with an objective to analyze the trends of sleep-related breathing disorders in patients with systemic essential hypertension.

\section{Methods}

\subsection{Study Design and Patient Population}

This was a prospective, single-center, observational study. Hypertensive patients who were attending LPS Institute of Cardiology and Dr. Murari Lal Chest Hospital at GSVM Medical College, Kanpur, India between December 2012 and July 2014 were selected randomly. Inclusion criteria included diagnosis of hypertension by Joint National Committee VII (JNC 7) criteria [5], i.e. systolic blood pressure $\geq 140 \mathrm{mmHg}$ and/or diastolic blood pressure $\geq 90 \mathrm{mmHg}$. Patients with secondary hypertension were excluded from the study. The selected patients were divided in to (a) stage I hypertensive group (i.e. systolic blood pressure $140-159 \mathrm{mmHg}$ and/or diastolic blood pressure $90-99 \mathrm{mmHg}$ ) and (b) stage II hypertensive group (systolic blood pressure $\geq 160$ $\mathrm{mmHg}$ and diastolic blood pressure $\geq 100 \mathrm{mmHg}$ ). At the time of enrollment, each patient was explained about the study procedure in detail. Subsequently, informed consent was obtained from all participants. The study protocol was approved by the Institutional Ethical Committee prior to the commencement of the study. The study conferred the standards of good clinical practice and the Declaration of Helsinki.

\subsection{Data Collection}

All patients were asked to report age, gender, anthropometric measures (i.e. height, weight, BMI, and neck circumference), concurrent diseases (i.e. diabetes mellitus, hyperthyroidism, and chronic obstructive pulmonary disease), and sleep habits. Symptoms of sleep-disordered breathing (i.e. disruptive snoring, frequent awakenings, morning headaches, and excessive daytime sleepiness), and duration of sleep-disordered breathing symptoms were noted. Additionally, all patients completed the Epworth Sleepiness Scale (ESS) questionnaire.

A polysomnography was performed for all enrolled patients using Embla S4000 Medcare, Iceland. The system had Somnologica studio 3.3.2 software, electrodes and cables to record the electroencephalogram, electroo- 
culogram, electromyogram of the chin and bilateral tibialis anterior muscles, electrocardiogram. Airflow was measured using nasal flow meter. Oxygen saturation was measured using a pulse oximeter. Chest and abdominal respiratory movements were monitored by respiratory inductive thoracic and abdominal belts respectively. Snoring was recorded using snore microphones attached to the neck. Body position was monitored using bodyposition sensor.

All studies were analyzed using the criteria of Rechtschaffen and Kales [6], and in close concordance with scoring updates given by the American Academy of Sleep Medicine (AASM) [7]. Total sleep time and sleep latency time (i.e. time duration from the lights off to the onset) were noted. The traditional Rechtschaffen and Kales terminology for the 5 sleep stages (i.e. stages 1, 2, 3, 4, and rapid eye-movement (REM) sleep) was used in this study. Apneas were scored when there was a complete cessation of airflow or at least $90 \%$ drop in the peak thermal sensor excursion for at least $10 \mathrm{~s}$. Hypopneas were scored when there was a drop in nasal pressure signal excursion by at least $50 \%$ of baseline lasting at least for $10 \mathrm{~s}$ with a $\geq 3 \%$ desaturation from pre-event baseline. The Apnea Hypopnea Index (AHI), which is number of apnea-hypopnea events per hour, was determined after the exclusion of periods with movements, which were considered to be wake periods. Next morning, a $5 \mathrm{~mL}$ of venous blood sample was withdrawn from each participant to analyze the C-reactive protein levels. Analysis and interpretation of study findings was carried out by trained technicians and sleep physicians involved in the study.

\subsection{Statistical Methods}

Data were analyzed using the Statistical Package for Social Sciences (SPSS for Windows version 20.0; Chicago, IL, USA). Qualitative data are presented as frequency and percentage, while quantitative data are described as mean value \pm standard deviation (SD). The Chi-square test was used to compare qualitative data and the Student's t-test was used to compare quantitative data between the two groups i.e. stage I hypertensives and stage II hypertensives. $p$ value $<0.05$ was considered to indicate statistically significant difference between the two groups.

\section{Results}

\subsection{Baseline Demographics}

A total 51 patients (age: $45.7 \pm 13.43$ years) were investigated in the study. Of them, $30(58.9 \%)$ were males. The study population consisted $21(41.2 \%)$ patients of 20 - 40 years age group, $20(39.2 \%)$ patients of 41 - 60 years age group, and $10(19.6 \%)$ patients of $>60$ years age group. The mean BMI for overall patients was $27.3 \pm 4.7$ $\mathrm{kg} / \mathrm{m}^{2}$. In particular, $19(37.2 \%)$ patients had BMI of $<25 \mathrm{~kg} / \mathrm{m}^{2}, 19(37.3 \%)$ patients had BMI of $25-29.9$ $\mathrm{kg} / \mathrm{m}^{2}, 10(19.6 \%)$ patients had BMI of $30-34.9 \mathrm{~kg} / \mathrm{m}^{2}$, and $3(5.9 \%)$ patients had BMI $\geq 35 \mathrm{~kg} / \mathrm{m}^{2}$. Comorbidities such as diabetes mellitus, hyperthyroidism and chronic obstructive pulmonary disease were identified in 9 $(17.6 \%), 3(5.9 \%)$ and, $3(5.9 \%)$ patients respectively.

Of 51 patients analyzed in the study, 36 (70.6\%) had stage I hypertension (mean blood pressure: 152/94 $\mathrm{mmHg}$ ) and 15 (29.4\%) had stage II hypertension (mean blood pressure: $168 / 104 \mathrm{mmHg}$ ). Of significance, more females had stage I hypertension than males (52.8\% females vs. $47.2 \%$ males). Contrary to this, stage II hypertension was more frequent among males than among females (86.7\% males vs. 13.3\% females). The baseline demographics and overall outcomes of sleep-disordered breathing vs. stage of hypertension are presented in Table 1 .

\subsection{Symptoms of Sleep-Disordered Breathing vs. Stage of Hypertension}

Nearly $50 \%$ of patients reported no symptoms of sleep-disordered breathing. Stage I hypertensive group had more asymptomatic patients as compared to stage II hypertensive group. Further, the symptoms of sleep-disordered breathing such as disruptive snoring, frequent awakenings, morning headaches, excessive daytime sleepiness and self-reported apneas were significantly more common among patients with stage II hypertension $(p<$ $0.05)$.

\subsection{Duration of Symptoms of Sleep-Disordered Breathing vs. Stage of Hypertension}

Symptom duration of more than 12 months was significantly associated with stage II hypertension $(p<0.05)$. 
Table 1. Study parameters of sleep-disordered breathing vs. stage of hypertension.

\begin{tabular}{|c|c|c|c|c|}
\hline Variable & $\begin{array}{l}\text { Overall patients } \\
\quad(\mathbf{n}=\mathbf{5 1})\end{array}$ & $\begin{array}{c}\text { Stage I } \\
\text { hypertensives } \\
(\mathbf{n}=\mathbf{3 6})\end{array}$ & $\begin{array}{c}\text { Stage II } \\
\text { hypertensives } \\
(\mathrm{n}=15)\end{array}$ & $p$ value $^{\mathrm{a}}$ \\
\hline \multicolumn{5}{|c|}{ Gender } \\
\hline Male, n (\%) & $30(58.8)$ & $17(47.2)$ & $13(86.7)$ & \multirow{2}{*}{$<0.05$} \\
\hline Female, n (\%) & $21(41.2)$ & $19(52.8)$ & $2(13.3)$ & \\
\hline Systolic blood pressure in $\mathrm{mmHg}$, mean $\pm \mathrm{SD}$ & $156.7 \pm 2$ & $152 \pm 3$ & $168 \pm 5$ & $<0.05$ \\
\hline Diastolic blood pressure in $\mathrm{mmHg}$, mean $\pm \mathrm{SD}$ & $96.9 \pm 3$ & $94 \pm 5$ & $104 \pm 6$ & $<0.05$ \\
\hline \multicolumn{5}{|c|}{ Symptoms of sleep-disordered breathing } \\
\hline No symptoms, n (\%) & $24(47.1)$ & $20(55.5)$ & $4(26.7)$ & $<0.05$ \\
\hline Disruptive snoring, $\mathrm{n}(\%)$ & $22(43.1)$ & $13(36.1)$ & $9(60.0)$ & $<0.05$ \\
\hline Frequent awakenings, $\mathrm{n}(\%)$ & $10(19.6)$ & $6(16.7)$ & $4(26.7)$ & $<0.05$ \\
\hline Morning headaches, $\mathrm{n}(\%)$ & $18(35.3)$ & $10(27.8)$ & $8(53.3)$ & $<0.05$ \\
\hline Excessive daytime sleepiness, n (\%) & $11(21.6)$ & $6(16.7)$ & $5(33.3)$ & $<0.05$ \\
\hline Self-reported apneas, n (\%) & $6(11.8)$ & $2(5.6)$ & $4(26.7)$ & $<0.05$ \\
\hline \multicolumn{5}{|c|}{ Duration of symptoms of sleep-disordered breathing } \\
\hline Asymptomatic, n (\%) & $24(47.1)$ & $20(55.5)$ & $4(26.7)$ & 0.05 \\
\hline$<6$ months, $\mathrm{n}(\%)$ & $5(9.8)$ & $4(11.1)$ & $1(6.7)$ & $<0.05$ \\
\hline 6 - 12 months, $\mathrm{n}(\%)$ & $9(17.6)$ & $5(13.9)$ & $4(26.7)$ & n.s. \\
\hline$>12$ months, $\mathrm{n}(\%)$ & $13(25.5)$ & $7(19.4)$ & $6(40.0)$ & $<0.05$ \\
\hline \multicolumn{5}{|c|}{ Epworth Sleepiness Scale (ESS) score } \\
\hline $0-10$ [No chances of dozing], $\mathrm{n}(\%)$ & $25(49.0)$ & $21(58.3)$ & $4(26.7)$ & n.s. \\
\hline $11-14$ [Slight chances of dozing], $\mathrm{n}(\%)$ & $13(25.5)$ & $8(22.2)$ & $5(33.3)$ & n.s. \\
\hline 15 - 18 [Moderate chances of dozing], $\mathrm{n}(\%)$ & $7(13.7)$ & $4(11.1)$ & $3(20.0)$ & n.s. \\
\hline 19 - 24 [High chances of dozing], n (\%) & $6(11.8)$ & $3(8.3)$ & $3(20.0)$ & $<0.05$ \\
\hline Total sleep time in minutes, mean $\pm \mathrm{SD}$ & $282.6 \pm 32.6$ & $288.5 \pm 37.9$ & $268.3 \pm 27.9$ & n.s. \\
\hline Sleep latency time in minutes, mean $\pm \mathrm{SD}$ & $12.4 \pm 0.90$ & $12.5 \pm \_0.80$ & $12.2 \pm 0.86$ & n.s. \\
\hline REM sleep latency time in minutes, mean $\pm \mathrm{SD}$ & $111.2 \pm 0.8$ & $109.9 \pm 0.78$ & $114.4 \pm 0.67$ & $<0.05$ \\
\hline Snore time in minutes, mean $\pm \mathrm{SD}$ & $95.9 \pm 32.1$ & $90.8 \pm 32.1$ & $108.6 \pm 29.5$ & $<0.05$ \\
\hline \multicolumn{5}{|c|}{ Apnea Hypopnea Index (AHI) score } \\
\hline$<5$ events/hour, $\mathrm{n}(\%)$ & $14(27.5)$ & $11(30.5)$ & $3(20.0)$ & n.s. \\
\hline $5-<15$ events/hour, $\mathrm{n}(\%)$ & $25(49.0)$ & $19(52.8)$ & $6(40.0)$ & n.s. \\
\hline $15-<30$ events/hour, $\mathrm{n}(\%)$ & $9(17.6)$ & $5(13.9)$ & $4(26.7)$ & n.s. \\
\hline$\geq 30$ events/hour, $\mathrm{n}(\%)$ & $3(5.9)$ & $1(2.8)$ & $2(13.3)$ & n.s. \\
\hline $\mathrm{AHI}$ (events per hour), mean $\pm \mathrm{SD}$ & $10.08 \pm 7.4$ & $8.48 \pm 5.8$ & $13.9 \pm 9.5$ & n.s. \\
\hline Patients with nocturnal oxygen desaturation, $\mathrm{n}(\%)$ & $17(33.3 \%)$ & $9(25.0)$ & $8(53.3)$ & n.s. \\
\hline Lowest oxygen saturation levels, mean $\pm \mathrm{SD}$ & $89.7 \pm 6.0$ & $90.9 \pm 4.8$ & $85.6 \pm 7.0$ & $<0.05$ \\
\hline $\mathrm{C}$-reactive protein levels in $\mathrm{mg} / \mathrm{dL}$, mean $\pm \mathrm{SD}$ & $0.81 \pm 0.08$ & $0.73 \pm 0.06$ & $0.99 \pm 0.1$ & $<0.05$ \\
\hline
\end{tabular}

${ }^{\mathrm{a}}$ For stage I vs. stage II hypertensives; n.s.: non-significant. 
Similarly, the frequency of patients with 6 - 12 month duration of symptoms was higher among stage II hypertensives than that among stage I hypertensives, but the difference was not significant. Conversely, the frequency of patients with $<6$ month duration of symptoms was higher among stage I hypertensives than that among stage II hypertensives $(p<0.05)$. Thus, the longer duration of symptoms was more common in patients with stage II hypertension, especially if the duration is more than 12 months.

\subsection{ESS Score vs. Stage of Hypertension}

Almost half the patients had no chances of dozing as displayed by normal ESS score (i.e. 0 - 10) and almost one quarter of the patients had slight chance of dozing as displayed by the ESS scores of $11-14$. Rest of the quarter patients had moderate to high ESS scores with more chances of dozing. Evidently, patients with stage II hypertension had higher scores for all sub-categories of ESS with $>10$ mean score indicating excessive daytime sleepiness when compared to that in patients with stage I hypertension. However, the difference was significant only in the group with mean ESS score of 19 - 24, which had the highest chance of dozing $(p<0.05)$.

\subsection{Total Sleep Time vs. Stage of Hypertension}

The overnight polysomnography test revealed that the average total sleep time in overall hypertensive patients was 282.6 minutes indicating sleep deprivation as the normal sleep time should be at least $6 \mathrm{~h}$. Further, the average total sleep time was significantly lesser among stage II hypertensives than that among stage I hypertensives $(p<0.05)$.

\subsection{Sleep Latency Time vs. Stage of Hypertension}

The sleep latency time, i.e. time duration from the lights off to the onset of NREM sleep, was comparable between both the groups. However, the REM sleep latency time was significantly higher among stage II hypertensives than that among stage I hypertensives $(p<0.05)$.

\subsection{Snoring Time vs. Stage of Hypertension}

The mean snore time was $90.8 \pm 32.1$ and $108.6 \pm 29.5$ minutes among stage I and stage II hypertensive patients respectively. It was significantly higher among stage II hypertensives as compared to stage I hypertensives $(p<$ $0.05)$.

\subsection{Sleep Architecture vs. Stage of Hypertension}

Percent duration of sleep in all stages i.e. N1, N2, N3 and REM, was almost similar between hypertensive patients of both stages (Figure 1). Maximum percentage of time was spent in N2 sleep followed by that in N3. However, REM sleep duration was found to be less than normal in both groups of patients.

\subsection{AHI Scores vs. Stage of Hypertension}

Out of 51 hypertensive patients included in the study, nearly $25 \%$ of patients had AHI $<5$ per hour, while almost $75 \%$ of the patients had AHI $<15$ per hour. Remaining subjects had higher AHI scores. The number of patients with AHI score of $\geq 30$ was very less in both stages of hypertension (i.e. one patient in the stage I hypertensive group and two patients in the stage II hypertensive group). Majority of patients in the respective sub-category of AHI $<5$ and AHI $5-<15$ belonged to stage I hypertensive group, while those with AHI 15 $<30$ and AHI $\geq 30$ had stage II hypertension. The differences in all sub-categories of AHI values between the two groups were statistically non-significant. Similarly, mean AHI was non-significantly higher among stage II hypertensives than in stage I hypertensives

\subsection{Nocturnal Oxygen Desaturation vs. Stage of Hypertension}

One-third of enrolled patients exhibited nocturnal oxygen desaturation. Of significance, more than half of patients with stage II hypertension had nocturnal oxygen desaturation as compared to the quarter of patients with stage I hypertension. However, the difference was statistically non-significant. On the other hand, the value of 


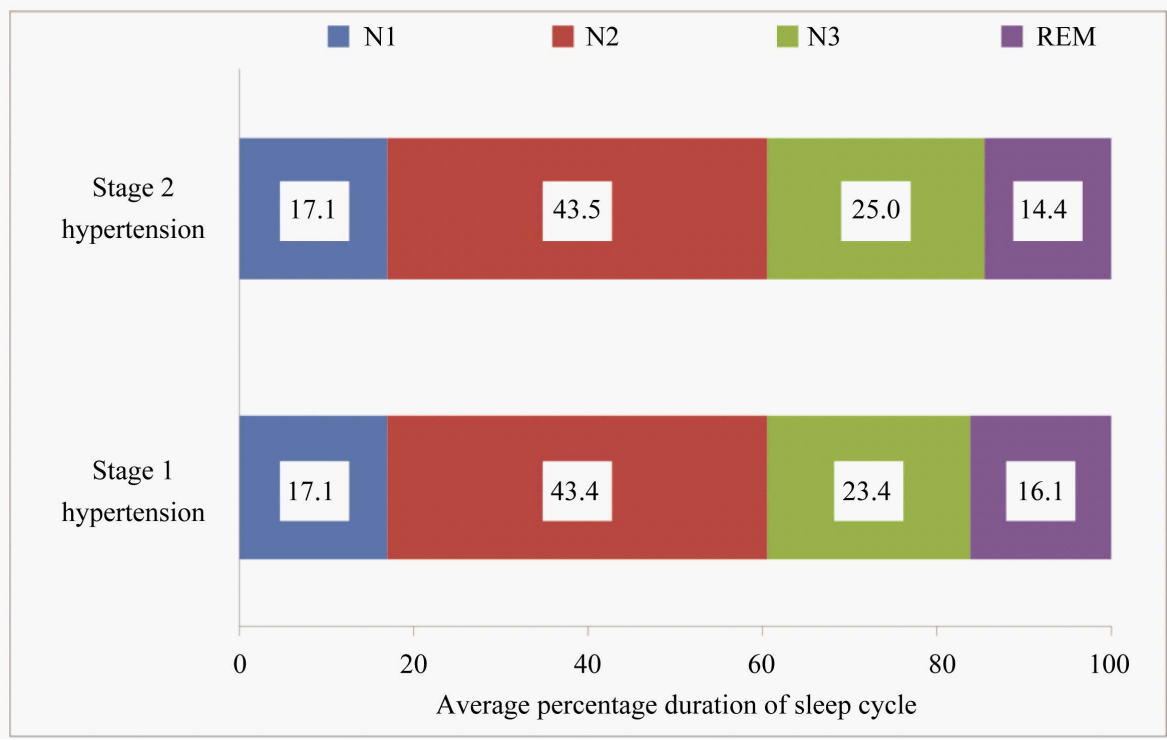

Figure 1. Average percentage duration of sleep stages vs. stage of hypertension.

average lowest oxygen saturation was significantly lesser among stage II hypertensives as compared to stage I hypertensives $(p<0.05)$.

\subsection{Mean Lowest Oxygen Saturation and Neck Circumference in Relation to AHI}

The mean lowest oxygen saturation decreased proportionately with increasing AHI and the differences were significant $(p<0.05$, Table 2). Similarly, the mean neck circumference of the subjects was higher with greater AHI scores $(p<0.05$, Table 2$)$.

\subsection{C-Reactive Protein Levels in Relation to AHI vs. Stage of Hypertension}

The mean C-reactive protein levels in both, stage I and II hypertensives, as well as in overall patients increased significantly with increase in the AHI values (Figure 2). The mean C-reactive protein levels were significantly higher in the stage II hypertensive group in all subgroups of patients with $\mathrm{AHI}<30(p<0.05)$.

\section{Discussion}

In this study, we evaluated sleep-related breathing disorders in 51 patients of essential hypertension. Majority of patients enrolled in our study were of age groups $<60$ years $(80.4 \%)$ and had BMI $>25 \mathrm{~kg} / \mathrm{m}^{2}(62.8 \%)$. BMI is considered as an important individual risk factor for sleep-related breathing disorder, and high BMI reported in our study warrants attention. Among the hypertensive patients enrolled in the present study, the frequency ratio of stage I hypertension and stage II hypertension was 7:3. A significant male predominance was observed with stage II hypertension.

Sleep-disordered breathing is one of the major determinants of hypertension. The casual relationship between sleep-disordered breathing and hypertension has been supported by both epidemiological and population-based studies [8]-[12]. It has been established that the association between sleep-related breathing and hypertension is largely influenced by the age of the patient. The effect of sleep-related breathing disorder on hypertension is more pronounced in younger and middle aged men than in those above 60 years [3].

The importance of investigating the association between sleep-related breathing disorders and cardiovascular disease is stressed by reports of a high prevalence of sleep apnea among patients with hypertension and coronary artery disease, as well as an increased mortality in patients with sleep apnea [1]-[3] [10]-[14]. Whether this is a true causal relationship or whether it is partly or completely caused by confounding is still a matter of debate. In our study, nearly $50 \%$ of enrolled hypertensives patients reported no symptoms of sleep-disordered breathing. We also observed that sleep-disordered breathing was more common and was more severe in majority of patients with stage II hypertension as compared to that in patients with stage I hypertension. 
Table 2. Mean lowest oxygen saturation and neck circumference in relation to AHI.

\begin{tabular}{cccc}
\hline & Number of patients & $\begin{array}{c}\text { Mean lowest oxygen } \\
\text { saturation (\%) }\end{array}$ & $\begin{array}{c}\text { Mean neck } \\
\text { circumference (cm) }\end{array}$ \\
\hline AHI $<5$ & 14 & $93.5 \pm 2.2$ & $34.7 \pm 4.7$ \\
AHI $5-<15$ & 25 & $89.3 \pm 5.6$ & $36.7 \pm 4.0$ \\
AHI $15-<30$ & 9 & $84.7 \pm 5.3$ & $38.1 \pm 5.4$ \\
AHI $\geq 30$ & 3 & $79.3 \pm 5.0$ & $41.7 \pm 3.1$ \\
Overall patients & 51 & $89.7 \pm 6.0$ & $36.6 \pm 4.6$ \\
\hline
\end{tabular}

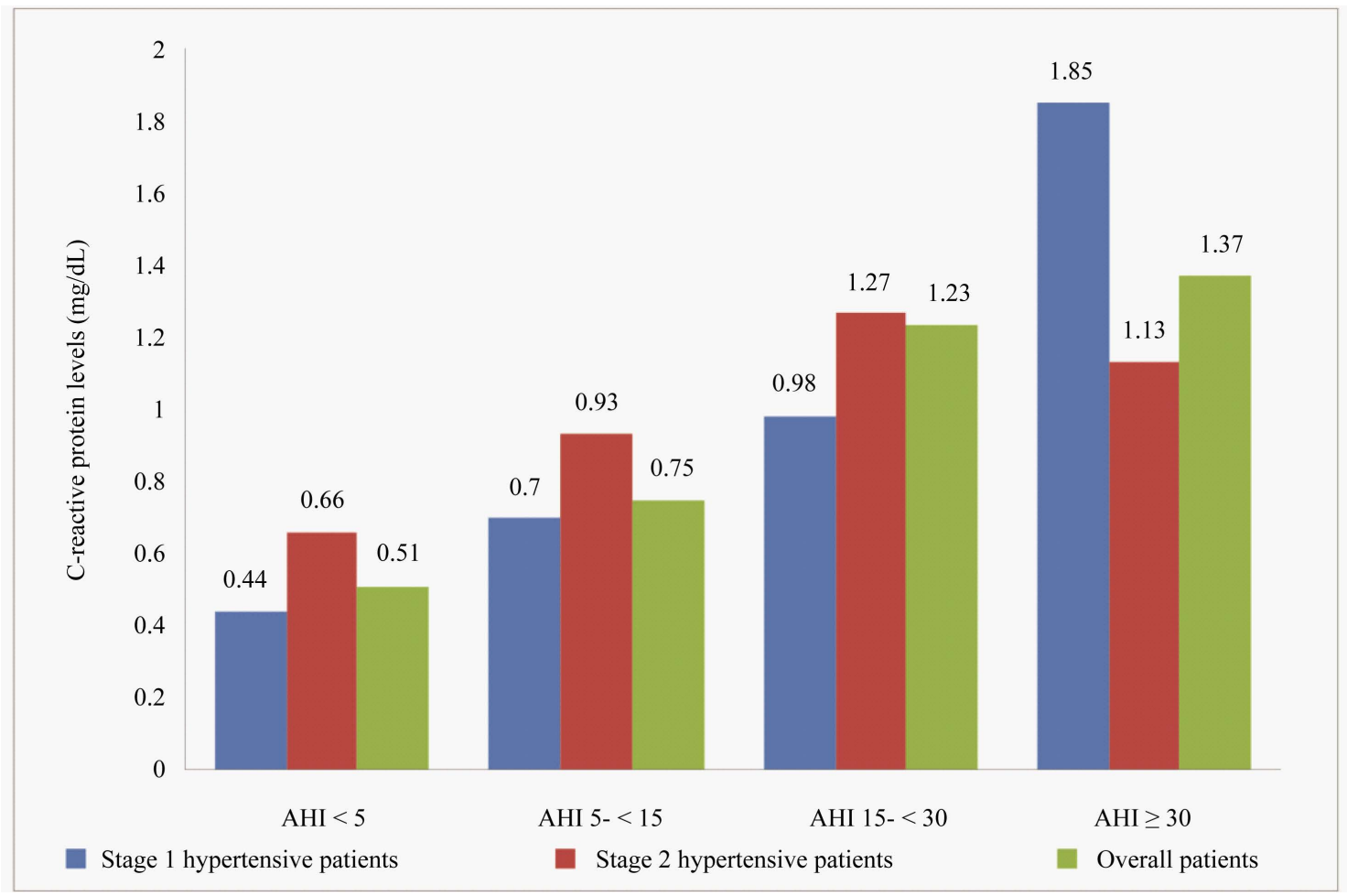

Figure 2. Mean C-reactive protein levels in relation to AHI and stage of hypertension.

Overnight polysomnography test revealed that patients with stage II hypertension had shorter total sleep time and longer snore time as compared to that in patients with stage I hypertension. It has been identified that sleep durations of $\leq 5$ hours per night was associated with a significantly increased risk of hypertension (hazard ratio, 2.10) [2]. In our study, the mean total sleep duration in overall patients was $<5$ hours, which could be a significant risk factor for hypertension. Further, our findings indicated that AHI values, nocturnal oxygen desaturation, and the mean C-reactive protein levels increased with the increase in severity of hypertension. This is also in line with several other studies of similar context [15].

It has been established that the AHI scores are correlated with the external neck circumference. [16] Our study was in concordance with this finding as AHI scores increased with mean neck circumference of the subjects included in our study. Similarly, we observed a proportionate decrease in the mean lowest oxygen saturation with increasing AHI scores. Of significance, our study indicated that the sleep architecture was similar in both stages of hypertension.

Earlier, studies have shown that mean blood pressure and the severity of hypertension increase with the severity of the sleep-related breathing disorder [17] [18]. In such studies, the comparative groups comprised normotensive patients, hypertensive patients, and drug-resistant hypertensive patients. To the best of our knowledge, till date no study had compared the presence and severity of the sleep-related breathing disorder in stage I and 
stage II hypertensive patients. Hence, findings of our study are of clinical significance to identify the role of sleep-related breathing disorder in the severity of hypertension.

Taking into account the financial and time constraint, the sample size was restricted to about 50 patients in our study. This can be considered as a major limitation of the study as generalization of the study findings may not be applicable due to small sample size. In this regard, a study with large sample size can be planned to overcome this limitation. Absence of stringent exclusion criteria could be considered as another limitation as we adopted no measures to control these extraneous variables. However, our study cohort was selected randomly and it should be considered as a representative of real-world patients.

\section{Conclusion}

In conclusion, our study reported that about $47 \%$ of patients with systemic essential hypertension displayed no symptoms of sleep-related breathing disorder. Comparison between stage I and stage II hypertensive groups revealed that the symptoms of sleep-related breathing disorder were more common and more severe among stage II hypertensives. To the best of our knowledge, the present investigation was a rare study that compared the trends of sleep-related breathing disorder between stage I and stage II hypertensive patients. Further large-scale studies are warranted to confirm the findings, which may help understand the pathophysiology and improve the management of hypertensive patients with sleep-related breathing disorders.

\section{References}

[1] Peppard, P.E., Young, T., Palta, M. and Skatrud, J. (2000) Prospective Study of the Association between Sleep-Disordered Breathing and Hypertension. The New England Journal of Medicine, 342, 1378-1384. http://dx.doi.org/10.1056/NEJM200005113421901

[2] Gangwisch, J.E., Heymsfield, S.B., Boden-Albala, B., Buijs, R.M., Kreier, F., Pickering, T.G., et al. (2006) Short Sleep Duration as a Risk Factor for Hypertension: Analyses of the First National Health and Nutrition Examination Survey. Hypertension, 47, 833-839. http://dx.doi.org/10.1161/01.hyp.0000217362.34748.e0

[3] Haas, D.C., Foster, G.L., Nieto, F.J., Redline, S., Resnick, H.E., Robbins. J.A., et al. (2005) Age-Dependent Associations between Sleep-Disordered Breathing and Hypertension: Importance of Discriminating between Systolic/Diastolic Hypertension and Isolated Systolic Hypertension in the Sleep Heart Health Study. Circulation, 111, 614-621. http://dx.doi.org/10.1161/01.CIR.0000154540.62381.CF

[4] Silverberg, D.S. and Oksenberg, A. (2001) Are Sleep-Related Breathing Disorders Important Contributing Factors to the Production of Essential Hypertension? Current Hypertension Reports, 3, 209-215. http://dx.doi.org/10.1007/s11906-001-0040-8

[5] Chobanian, A.V., Bakris, G.L., Black, H.R., Cushman, W.C., Green, L.A., Izzo, J.L., et al. (2003) The Seventh Report of the Joint National Committee on Prevention, Detection, Evaluation, and Treatment of High Blood Pressure: The JNC 7 Report. The Journal of the American Medical Association, 289, 2560-2572. http://dx.doi.org/10.1001/jama.289.19.2560

[6] Kales, A. and Rechtschaffen, A., University of California, Los Angeles, Brain Information Service \& NINDB Neurological Information Network (U.S.) (1968) A Manual of Standardized Terminology, Techniques and Scoring System for Sleep Stages of Human Subjects. In: Rechtscaffen, A. and Kales, A., Eds., Neurological Information Network, National Institute of Neurological Diseases and Blindness, Bethesda.

[7] Berry, R.B., Budhiraja, R., Gottlieb, D.J., Gozal, D., Iber, C., Kapur, V.K., et al. (2012) Rules for Scoring Respiratory Events in Sleep: Update of the 2007 AASM Manual for the Scoring of Sleep and Associated Events. Deliberations of the Sleep Apnea Definitions Task Force of the American Academy of Sleep Medicine. Journal of Clinical Sleep Medicine, 8, 597-619.

[8] Kapur, V.K., Resnick, H.E., and Gottlieb, D.J., Sleep Heart Health Study Group (2008) Sleep Disordered Breathing and Hypertension: Does Self-Reported Sleepiness Modify the Association? Sleep, 31, 1127-1132.

[9] Grote, L., Ploch, T., Heitmann, J., Knaack, L., Penzel, T. and Peter, J.H. (1999) Sleep-Related Breathing Disorder Is an Independent Risk Factor for Systemic Hypertension. American Journal of Respiratory and Critical Care Medicine, 160, 1875-1882. http://dx.doi.org/10.1164/ajrccm.160.6.9811054

[10] Nieto, F.J., Young, T.B., Lind, B.K., Shahar, E., Samet, J.M., Redline, S., et al. (2000) Association of Sleep-Disordered Breathing, Sleep Apnea, and Hypertension in a Large Community-Based Study. Sleep Heart Health Study. The Journal of the American Medical Association, 283, 1829-1836. http://dx.doi.org/10.1001/jama.283.14.1829

[11] Lavie, P., Herer, P. and Hoffstein, V. (2000) Obstructive Sleep Apnoea Syndrome as a Risk Factor for Hypertension: 
Population Study. BMJ, 320, 479-482. http://dx.doi.org/10.1136/bmj.320.7233.479

[12] Bixler, E.O., Vgontzas, A.N., Lin, H.M., Ten Have, T., Leiby, B.E., Vela-Bueno, A., et al. (2000) Association of Hypertension and Sleep-Disordered Breathing. Archives of Internal Medicine, 160, 2289-2295. http://dx.doi.org/10.1001/archinte.160.15.2289

[13] Quan, S.F. and Gersh, B.J., National Center on Sleep Disorders Research, National Heart, Lung, and Blood Institute (2004) Cardiovascular Consequences of Sleep-Disordered Breathing: Past, Present and Future. Report of a Workshop from the National Center on Sleep Disorders Research and the National Heart, Lung, and Blood Institute. Circulation, 109, 951-957. http://dx.doi.org/10.1161/01.CIR.0000118216.84358.22

[14] Cintra, F.D., Tufik, S., Paola, Ad., Feres, M.C., Melo-Fujita, L., Oliveira, W., et al. (2011) Cardiovascular Profile in Patients with Obstructive Sleep Apnea. Arquivos Brasileiros de Cardiologia, 96, 293-299. http://dx.doi.org/10.1590/S0066-782X2011005000030

[15] Kumar, R. and Kochhar, S. (2013) C-Reactive Protein and Severity of Hypertension. Journal of Advance Researches in Biological Sciences, 5, 331-335.

[16] Katz, I., Stradling, J., Slutsky, A.S., Zamel, N. and Hoffstein, V. (1990) Do Patients with Obstructive Sleep Apnea Have Thick Necks? American Review of Respiratory Disease, 141, 1228-1231. http://dx.doi.org/10.1164/ajrccm/141.5_Pt_1.1228

[17] Grote, L., Hedner, J. and Peter, J.H. (2001) Mean Blood Pressure, Pulse Pressure and Grade of Hypertension in Untreated Hypertensive Patients with Sleep-Related Breathing Disorder. Journal of Hypertension, 19, 683-690. http://dx.doi.org/10.1097/00004872-200104000-00004

[18] Sjöström, C., Lindberg, E., Elmasry, A., Hägg, A., Svärdsudd, K. and Janson, C. (2002) Prevalence of Sleep Apnoea and Snoring in Hypertensive Men: A Population Based Study. Thorax, 57, 602-607. http://dx.doi.org/10.1136/thorax.57.7.602 